\title{
Erratum to "Frames for Weighted Shift-Invariant Spaces", Mediterr. J. Math., DOI:10.1007/s00009-011-0155-3
}

\author{
Stevan Pilipović and Suzana Simić
}

Lemma $5.1 \mathrm{~b}$ ), Theorem 5.2 and Theorem $5.4 \mathrm{~b}$ ) as stated in [1] are not true. However, they hold only in some special cases included in the next theorem.

Theorem 0.1. a) Let $\theta \in C_{0}^{\infty}(\mathbb{R})$ be a positive function such that $\theta(x)>0$, $x \in A, A \subset[-\pi, \pi]$, and $\operatorname{supp} \theta \subseteq[-\pi, \pi]$. Moreover, let

$$
\widehat{\phi}_{k}(\xi)=\theta(\xi+k \pi), \quad k \in \mathbb{Z},
$$

and $\Phi=\left(\phi_{i}, \phi_{i+1}, \ldots, \phi_{i+r}\right)^{T}, i \in \mathbb{Z}, r \in \mathbb{N}$.

Then the rank of the matrix $[\widehat{\Phi}(\xi+2 j \pi)]_{j \in \mathbb{Z}}$ is not a constant function on $\mathbb{R}$ and it depends on $\xi \in \mathbb{R}$.

b) Let $\theta \in C_{0}^{\infty}(\mathbb{R})$ be a positive function such that

$$
\theta(x)>0, \quad x \in(-\pi-\varepsilon, \pi+\varepsilon),
$$

and supported by $[-\pi-\varepsilon, \pi+\varepsilon]$ where $0<\varepsilon<1 / 4$. Moreover, let

$$
\widehat{\phi}_{i}(\xi)=\theta\left(\xi+k_{i} \pi\right), \quad k_{i} \in \mathbb{Z}, i=1,2, \ldots, r, r \in \mathbb{N},
$$

and $\Phi=\left(\phi_{1}, \phi_{2}, \ldots, \phi_{r}\right)^{T}$.

1) If $\left|k_{2}-k_{1}\right|=2$ and $\left|k_{i}-k_{j}\right| \geq 2$ for different $i, j \leq r$, then the rank of the matrix $[\widehat{\Phi}(\xi+2 j \pi)]_{j \in \mathbb{Z}}$ is a constant function on $\mathbb{R}$ and equals $r$.

2) If $\left|k_{2}-k_{1}\right|=2$ and, at least for $k_{i_{1}}$ and $k_{i_{2}}$, it holds that $\left|k_{i_{1}}-k_{i_{2}}\right|=$ 1 , where $1 \leq i_{1}, i_{2} \leq r$, then the rank of the matrix $[\widehat{\Phi}(\xi+2 j \pi)]_{j \in \mathbb{Z}}$ is a non-constant function on $\mathbb{R}$.

The proof of the theorem, together with other additional results, will appear in a forthcoming paper, a preliminary version of which appears in ArXiv (2011) arXiv:1109.3285.

The online version of the original article can be found under doi:10.1007/s00009-011-0155-3. 


\title{
Reference
}

[1] S. Pilipović and S. Simić, Frames for weighted shift-invariant spaces, to appear in Mediterr. J. Math., DOI:10.1007/s00009-011-0155-3.

\author{
Stevan Pilipović \\ Department of Mathematics and Informatics, Faculty of Science \\ University of Novi Sad \\ Trg Dositeja Obradovica 4, 21000 Novi Sad \\ Serbia \\ e-mail: stevan.pilipovic@dmi.uns.ac.rs \\ Suzana Simić \\ Department of Mathematics and Informatics, Faculty of Science \\ University of Kragujevac \\ Radoja Domanovića 12, 34000 Kragujevac \\ Serbia \\ e-mail: suzanasimic@kg.ac.rs
}

